Pneumologe 2017 $\cdot 14: 197$

DOI 10.1007/s10405-017-0121-2

Online publiziert: 8. Juni 2017

(c) Springer Medizin Verlag GmbH 2017

CrossMark

Schon früh hat die Deutsche Gesellschaft für Pneumologie und Beatmungsmedizin die große Bedeutung der Wechselwirkungen zwischen Herz und Lunge erkannt. So wurde vor fast 25 Jahren die wissenschaftliche Sektion 6 ins Leben gerufen, deren Schwerpunkt die klinisch relevanten Zusammenhänge zwischen den beiden Organsystemen sind.

\section{I) Die neuen therapeutischen Möglichkeiten berücksichtigen die kardiorespiratorische Interaktion}

Durch das zunehmende pathophysiologische Verständnis wurden in den letzten Jahren neue Verfahren in der nichtinvasiven und invasiven Diagnostik von Herz- und Lungenerkrankungen entwickelt. Davon profitieren die Patienten der Pneumologie und der Kardiologie gleichermaßen. Die neuen therapeutischen Möglichkeiten unter Einschluss des körperlichen Trainings als nichtmedikamentöser Therapieoption berücksichtigen die kardiorespiratorische Interaktion, um die bestmöglichen Effekte zu erzielen.

Die 5 Beiträge im vorliegenden Themenheft stellen dem Leser ausgewählte Aspekte aus dem Spektrum der kardiorespiratorischen Interaktion praxisnah dar.

Die Limitationen der früheren Trennung zwischen Morphologie und Funktion des Pulmonalkreislaufs sind durch die Möglichkeiten der modernen Bildgebungsverfahren überwunden. Erst durch die Kombination von Ultraschall, Röntgenstrahlen oder Magnetresonanz lassen sich das rechte Herz und die nachgeschal-

\author{
F. J. Meyer ${ }^{1} \cdot \mathrm{H}$. Wilkens ${ }^{2} \cdot \mathrm{H}$. Worth ${ }^{3}$ \\ ' Lungenzentrum München (LZM Bogenhausen-Harlaching), Städtisches Klinikum München, München, \\ Deutschland \\ ${ }^{2}$ Medizinische Klinik V, Pneumologie, Allergologie und Beatmungsmedizin, Universitätsklinikum des \\ Saarlandes, Homburg, Deutschland \\ ${ }^{3}$ Facharztforum Fürth, Fürth, Deutschland
}

\title{
Kardiorespiratorische Interaktion
}

tete Blutstrombahn heute immer exakter beurteilen.

Diese verbesserte Bildgebung kommt auch den Patienten mit Lungenarterienembolie zu raschen Diagnosestellung und Einleitung der adäquaten Therapie zugute. Die Nachsorge und damit Früherkennung einer (persistierenden) Rechtsherzbelastung im Verlauf nach Embolie gewinnt angesichts der verbesserten konservativen und chirurgischen Therapieoptionen immer mehr an Bedeutung.

Die Bedeutung von Diabetes mellitus für kardiovaskuläre Erkrankungen und auch die Auswirkungen auf die Lungenfunktion sind seit langem bekannt. In Bezug auf Lungenerkrankungen werden diese Zusammenhänge im klinischen Alltag jedoch häufig wenig berücksichtigt. Da die Lunge oftmals signifikanten Einfluss auf den Zustand und die Leistungsfähigkeit von Patienten mit einer primär kardiovaskulären Diagnose bzw. Therapie hat, sollte der behandelnde Arzt zur optimalen Versorgung seiner Patienten diese Zusammenhänge kennen. Darauf fokussieren eigene Beiträge in diesem Themenheft.

\section{》) Nicht alle Trainingsangebote sind für jeden Menschen sinnvoll oder unbedenklich}

Abschließend wird ein geradezu gesellschaftliches Phänomen beleuchtet: Gesunde und Patienten werden heutzutage vielerorts zur körperlichen Tätigkeit angehalten. Aber nicht alle Trainingsangebote sind für jeden Menschen sinnvoll oder unbedenklich. Vielmehr sind die individuelle Auswahl der richtigen Bewegungsform im richtigen Umfang sowie deren adäquates Monitoring Voraussetzungen für die gewünschten positiven Effekte.

Die Wechselwirkungen zwischen Herz und Lunge sind oftmals komplex. Dieses Themenheft vermittelt hoffentlich etwas von der Faszination und der klinischen Bedeutung der kardiorespiratorischen Interaktion.

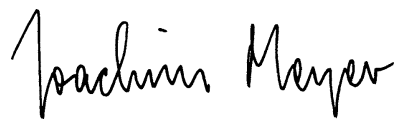

Prof. Dr. F. Joachim Meyer

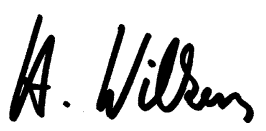

Prof. Dr. Heinrike Wilkens

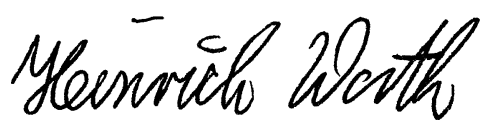

Prof. Dr. Heinrich Worth

\section{Korrespondenzadresse}

Prof. Dr. F. J. Meyer

Lungenzentrum München (LZM BogenhausenHarlaching), Städtisches Klinikum München München, Deutschland

joachim.meyer@klinikum-muenchen.de

\section{Prof. Dr. H. Wilkens}

Medizinische Klinik V, Pneumologie, Allergologie und Beatmungsmedizin, Universitätsklinikum des Saarlandes 66421 Homburg, Deutschland Heinrike.Wilkens@uniklinikum-saarland.de

Interessenkonflikt. F.J. Meyer, H. Wilkens und $\mathrm{H}$. Worth geben an, dass kein Interessenkonflikt besteht. 